Published in final edited form as:

Curr Neurol Neurosci Rep. ; 17(11): 91. doi:10.1007/s11910-017-0798-y.

\title{
Treatment Advances in Spinal Muscular Atrophy
}

Diana Bharucha-Goebel ${ }^{1,2,{ }^{*}}$ and Petra Kaufmann ${ }^{3,{ }^{*}}$

${ }^{1}$ Department of Neurology, Children's National Medical Center, Washington, DC

${ }^{2}$ Neuromuscular and Neurogenetic Disorders of Childhoood Section (NNDCS)/NINDS/NIH, Bethesda, MD

${ }^{3}$ National Center for Advancing Translational Sciences (NCATS)/NIH, Bethesda, MD

\begin{abstract}
Purpose of Review-Spinal Muscular Atrophy (SMA) is a genetic disorder of motor neurons in the anterior horns of the spinal cord and brainstem that results in muscle atrophy and weakness. SMA is an autosomal recessive disease linked to deletions of the $S M N 1$ gene on chromosome 5q. Humans have a duplicate gene (SMN2) whose product can mitigate disease severity, leading to the variability in severity and age of onset of disease, and is therefore a target for drug development.
\end{abstract}

Recent Findings-Advances in preclinical and clinical trials have paved the way for novel therapeutic options for SMA patients, including many currently in clinical trials. In 2016, the first treatment for SMA has been approved in the US, an antisense-oligonucleotide that increases fulllength protein product derived from SMN2.

Summary-The approval of a first treatment for SMA and the rapid advances in clinical trials provide the prospect for multiple approaches to disease modification. There are several other promising therapeutics in different stages of development, based on approaches such as neuroprotection, or gene therapy.

\section{Keywords}

Spinal Muscular Atrophy; clinical trials; gene therapy; natural history; functional biomarkers; newborn screening; standard of care

\section{Introduction}

The term "Spinal Muscular Atrophy" (SMA) describes the underlying macropathology of the disease, atrophy and degeneration of the anterior horns in the spinal cord and brainstem resulting in progressive muscle atrophy and weakness. The condition was first described by

\footnotetext{
* The views expressed are those of the authors and do not reflect official NIH perspectives or policies.

Correspondence to: Diana Bharucha-Goebel.

Compliance with Ethics Guidelines

Conflict of Interest

Diana Bharucha-Goebel and Petra Kaufmann declare no conflict of interest.

Human and Animal Rights and Informed Consent

This article does not contain any studies with human or animal subjects performed by any of the authors.
} 
Guido Werdnig and Johann Hoffman in 1891, who described severe cases of onset in infancy [1]. SMA is the most common fatal genetic disease in infants with an incidence of about 1/6000-10,000 live births, out of which about 60\% develop SMA type 1, the most severe form of chromosome 5-associated SMA. The prognosis varies by severity, with SMA type 1 infants without disease specific treatment typically requiring ventilator or nutritional support in the first two years of life, and in the past rarely survived past age 2 years. Individuals with SMA type 2 typically have a normal life expectancy, as do those with SMA type 3 [1-3]. The diagnosis is clinically suggested by muscle atrophy, weakness, loss of reflexes, tongue fasciculations, tremor and denervation on EMG, and is confirmed by genetic testing showing (most typically) deletions in the Survival of Motor Neuron (SMNI) gene. This review will describe the mechanisms of disease, clinical phenotypes, therapeutic approaches, and care considerations.

\section{Pathophysiology}

SMA is characterized by a loss of lower motor neurons, that leads to muscle atrophy and wasting and clinically with weakness. Humans possess a unique gene, SMN2, that is similar to $S M N 1$ and can be present in multiple copies. However, $S M N 2$ differs from $S M N 1$ by a C to $\mathrm{T}$ substitution in exon 7 [3]. This substitution results in the exclusion of this exon and therefore an unstable transcript, leading to a very low yield (only about 10-15\%) of full length protein product. This is without clinical consequence in individuals who have at least one normal copy of $S M N 1$, but in patients with SMA who have deletions/mutations in $S M N 1$, the number of copies of $S M N 2$ impacts the disease severity (with the more copies of $S M N 2$ defining a milder phenotype). The SMN protein is found throughout the cytoplasm and nucleus, and it plays a role in spliceosomal small nuclear ribonuclear protein (snRNP) biogenesis and in pre-mRNA splicing[1]. SMN protein also exists in the axons of motor neurons. It is unclear exactly how reduced SMN levels cause SMA. There are several hypotheses as to how the absence of SMN results in SMA, and it is possible that these hypothesis are linked: 1) Disrupting the formation of snRNP's affects splicing of select genes that play a role in motor neuron circuitry; 2) Since SMN protein exists in the axons of motor neurons, there could be a disruption within the axons in SMA [1, 4]. Animal models have shown an early vulnerability of the neuromuscular junction. It is unclear if effects on the neuromuscular junction (NMJ) and also on muscle are purely secondary to failure of the terminal motor axons, or whether SMN deficiency has any primary pathology on NMJ and muscle. Clinically, motor neurons are not all equally affected during various stages of the disease course in a given patient. This difference is thought to be due to varying motor neuron vulnerability which may be influenced by: size, neuromuscular junction maturation pathway, and other variables [3]. Additional candidate mechanisms for disease pathophysiology include apoptosis, DNA repair, and ribosome and rRNA binding [3].

\section{Clinical Phenotype}

SMA is characterized clinically by progressive muscle atrophy and weakness, loss of reflexes, tongue fasciculations, tremor and denervation on EMG. Early in disease, facial muscles are relatively spared compared to muscle groups in the extremities and core/trunk muscles. Cognition is generally spared in patients with SMA, and often patients excel and 
have higher than average intelligence. The disease severity is categorized based on the highest motor milestone achieved (see Table 1). SMA type 1 describes infants with disease onset before 6 months of age who do not achieve the milestone of independent sitting. Infants with type 1 SMA usually appear normal in tone and strength at birth and subsequently develop weakness, respiratory distress, poor feeding, and low tone. These patients typically have a bell shaped chest due to poor chest wall expansion in the setting of relatively preserved diaphragmatic function [5]. SMA type 2 describes children with disease onset typically between 6 to 18 months of age who achieve the ability to sit independently, but do not achieve independent walking. Patients with type 2 SMA exhibit proximal weakness that is more severe in the lower extremities than upper extremities early on. Reflexes are typically absent. A fine tremor may appear on reach tasks. Scoliosis is common in this group of patients. A majority of patients with SMA type 2 survive into adulthood, but may require supportive care due to impaired swallowing and respiratory insufficiency. SMA type 3 describes individuals who are able to walk at some point in their lives, even if they subsequently lose independent ambulation. Onset is anywhere from 18 months to adulthood. This group has further been subdivided by patients with earlier onset (between 18 months and 3 years old) - type $3 a$ and those with later onset between ages 3 and 21 years old - type $3 b$. Patients often present with frequent falls, difficulty climbing steps and proximal weakness. There is a more severe form, SMA Type 0 , that is clinically defined by prenatal onset. Prenatal findings include decreased fetal movements, that may subsequently lead to joint contractures. At birth, these patients have severe hypotonia and weakness, tongue fasciculations, respiratory distress nearly always requiring respiratory assistance within hours of life, as well as bulbar weakness, autonomic dysfunction, and in some, congenital heart defects (septal defects). (Grotto, et al; 6) A milder form is sometimes referred to as SMA type 4, defined as individuals with onset in later childhood or in adulthood who are able to walk unassisted. The disease subtypes are defined as described above by their motor ability achieved, and are typically correlated with copy number of SMN2.

Efforts to better understand disease progression and define outcome measures in the natural history of the disease in the various subtypes of SMA have also paved the way for clinical trial readiness in SMA. Use of outcome measures in part depends on the subtype of disease. The Hammersmith Functional Motor Scale Expanded (HFMSE) is a motor function scale that has been specifically designed for SMA. Activities in this scale target items of functional relevance in this disease [7]. The Gross Motor Function Measure (GMFM) or the Motor Function Measure 32 (MFM32) are functional scales, with the MFM32 validated for patients with neuromuscular disease. The 6-minute walk test (6MWT) is an additional functional score that is applicable for patients who are independently ambulant. This test can measure exercise capacity and is sensitive to fatigue related changes in SMA [8-10]. Functional tests for infants with SMA include the Hammersmith Infant Neurological Exam part 2 (HINE-2) and the Children's Hospital of Philadelphia Infant Test of Neuromuscular Disorders (CHOP-INTEND) [8]. Quantitative muscle testing can be performed in children > 5 years old. The respiratory complications are a major part of the disease phenotype, especially in patients with Type 1 and Type 2 SMA. Evaluation of the utility of pulmonary function testing in SMA patients shows that it may better reflect disease state than muscle strength. However, PFTs are difficult to perform and can have variability with effort - 
factors which need to be taken into consideration when considering these as major clinical trial endpoints[9]. Assessments of quality of life can be ascertained through the peds QL (pediatric health-related QOL scale) [9]. Electrophysiologically, compound motor action potential (CMAP) amplitudes correlate to clinical severity and function. An additional marker, motor unit number estimation (MUNE), assesses the number of motor neurons or axons innervating a muscle or group of muscles[5]. Needle EMG and evaluation of motor unit action potentials and recruitment patterns can further help to show the extent of denervation and motor unit drop out. Quantitative muscle imaging and serum biomarkers have also been explored at potential outcome measures [11].

Setting the stage for clinical trials, several reports have described the natural history of the different types of SMA. For SMA type 1, nutritional and respiratory support prolonged life expectancy[12]. Overall, motor and respiratory function declined rapidly in the first two years of life, and most patients died or reached dependency on respiratory support by age two years [12-13]. For SMA types 2 and 3, disease progression as measured in terms of motor function and pulmonary function declined only slowly over time [14]. A study by Mercuri et al. evaluated the 12-month change in function as scored by the HFMSE in both ambulant and non-ambulant patients with Type 2 and 3 SMA. Their group found that baseline HFMSE scores were significantly different between ambulant patients (mean 53.6) and non-ambulant patients (mean 13.8). [15] When subdividing the ambulant and nonambulant groups, there was also a significant difference in the 12-month changes between the two groups. It has been through a wealth of coordinated natural history studies and investigations targeting physiologic biomarkers, measures of strength and function, as well as other disease relevant markers that has set the stage for the number of clinical trials in SMA, and specifically for the efficacy seen in the first approved treatment for SMA (see below).

\section{Therapeutic Approaches}

Since the discovery of the SMN gene in 1995, the development of animal models as well as targeted approaches to increase SMN protein levels has accelerated therapeutic development for SMA. The abilty to increase SMN expression through the use of small molecule therapy and with antisense oligonucleotide therapy was demonstrated in tissue culture, then in preclinical studies. Compounds were identified through high-throughput screens for candidate molecules that could increase SMN protein levels [1]. Histone deacetylase inhibitors such as valproic acid, phenylbutyrate, and trichostatin A activate the SMN2 promotor, driving increased full length SMN expression [1, 16]. HDAC inhibition is not specific to SMN, thus leading to a potential for side effects and potentially to dose limitations. The relationship between the number of $S M N 2$ copies and phenotypic severity has been recapitulated in mouse models where more $S M N 2$ copies mitigate severity, and was found to have favorable results in preclinical studies [1]. Animal models have also helped identify that there appears to be a therapeutic window, beyond which induction of SMN expression is less effective [17]. Approaches such as gene therapy and antisense oligonucleotide therapy, which showed robust improvements in the health of mice showed the greatest effects when delivered within the first postnatal days in mice (P0 - P3), but were less effective beyond P5. In a porcine model given gene transfer with scAAV9-SMN at the 
time of symptom onset, a marked improvement was still seen in the proximal weakness and in electrophysiological studies, suggesting that this window may be somewhat larger in patients [17]. While the animal models cannot fully predict the human SMA response to therapeutics or the therapeutic window for initiation of treatment, the concept of delivering or initiating a therapeutic strategy in the presymptomatic phase or early in the symptomatic phase would be predicted to have the greatest therapeutic benefit.This has now most recently been recapitulated in clinical trials with antisense oligonucleotide therapies and from early evidence in the gene delivery approach as well. The possibility of increasing full length protein by targeting SMN2 has resulted in the development of several therapeutic candidates, in addition to approaches such as gene therapy or neuroprotection.

\section{Clinical Trials}

Earlier clinical trials aimed at increasing full length SMN production from $S M N 2$ were focused on histone-deacetylase (HDAC) inhibitors and related mechanisms. Valproic acid, an HDAC inhibitor was shown to result in a $2-4$ fold increase in SMN protein in SMA patient derived fibroblasts, and subsequently showed increased median survival in SMA mouse models (with an increase in SMN protein in the spinal cord and improvements in muscle and neuron pathology). [16] The Carni-Val I clinical trial enrolled 61 patients aged 2-8 years with the ability to sit to receive valproic acid or placebo for 6 months. There was no improvement in motor function. $80 \%$ had adverse events, mostly weight gain, which was negatively related to motor function change [18]. Carni-Val 2 is a trial of 33 SMA type 3 aged 3-17 years with the ability to walk who received valproic acid or placebo or valproic acid for 12 months without associated change in motor function. $17 \%$ had a weight gain of more than 20\% [19]. Trichostatin A, a more potent HDAC inhibitor was tested in mice and showed increased weight gain, stabilization of motor function, and intact neuromuscular junctions even after treatment was discontinued. However, given the poor HDAC selectivity with trichostatin A, it was felt too toxic for chronic use to treat SMA [16]. Albuterol is a $\beta 2$ adrenergic receptor agonist used mostly for asthma, but has been shown to increase fulllength SMN transcript levels in severe SMA patient derived cell lines. A small trial in type II and type III SMA patients showed a statistically significant increase in muscle function; however, there has been no data from a larger placebo controlled trial to suggest the use of albuterol widely in clinical practice for SMA [16]. Hydroxyurea is an FDA approved compound used for certain solid tumors and for sickle cell anemia that had been considered for SMA. While early uncontrolled trials showed trends towards benefit, a subsequent controlled trial of hydroxyurea showed no benefit in 28 patients with SMA types 2 and 3 receiving hydroxyurea or placebo with monitoring of motor function, manual muscle testing, or full-length SMN mRNA [16].

Several other trials have attempted to repurpose therapeutics approved for other indications for the benefit of SMA. These trials have evaluated compounds including creatine, phenylbutyrate, gabapentin, thyrotropin releasing hormone, albuterol, riluzole, ceftriaxone, and aclarubicine [16, 21-21]. Agents such as riluzole and ceftriaxone require further studies to determine whether there is benefit for SMA patients. 
More recently, there have been newer therapeutic approaches, including additional small molecule approaches as well as oligonucleotide therapy and even gene replacement therapy (Figure 1).

Olexosime (Tro19622) is a compound in the cholesterol-oxime family that targets mitochondrial integrity and function in stressed cells. It promotes the survival of motor neurons under stress conditions in pre-clinical studies. In a study of 160 SMA type 2 and non-ambulatory type 3 patients aged 3-25 years at 22 sites in 7 countries, motor function was tested over 2 years of treatment with TRO19622 or placebo using the primary outcome measure, Motor Function Measure (MFM) [22]. Olesoxime seemed to be safe and well tolerated. The primary endpoint was not met, although secondary endpoints suggest that olesoxime might maintain motor function in patients with type 2 or type 3 SMA over a period of 24 months. [22

PTC Therapeutics identified a compound (RG7800) that selectively modulates SMN2 inclusion of exon 7 and they, in partnership with Roche, chemically optimized the compound as an orally bioavailable drug. A phase I single-ascending dose study revealed that the compound was safe and well tolerated in healthy volunteers. A randomized, placebo controlled Phase Ib/IIa clinical trial in adult and pediatric SMA patients began to enroll, but was suspended due to findings in long term preclinical studies that showed an unexpected eye condition. In January 2016, Roche initiated a phase I safety and pharmacokinetic trial in healthy volunteers of an alternate but similar compound RG7916. Currently, Roche has initiated two phase I/II studies to assess safety, tolerability, and pharmacokinetics of RG7916 in infants with type I SMA (FIREFISH - NCT 02913482) and in Type 2 and Type 3 SMA (SUNFISH - NCT 02908685) [16, 23]. These studies are both ongoing at the time of writing. An additional safety and pharmacokinetics study of R07034067 in children and adults who have previously been treated with a survival motor neuron2 (SMN2) targeting therapy (JEWELFISH -NCT03032172) is also underway.

Astellas in combination with Cytokinetics has developed CK-107/CK-2127107, a fast skeletal muscle troponin activator aimed to slow the rate of calcium release from the regulatory troponin complex of fast skeletal muscle fibers. This drug is targeted to increase skeletal muscle contractility and therefore enhance physical performance. This has been studied in Phase 1 clinical trials in healthy volunteers and is currently in a phase 2, double blind, placebo-controlled, multiple dose study aimed at studying the pharmacodynamic effect of CK-2127107 on skeletal muscle function and fatigability for patients with Type 2, Type 3, and Type 4 SMA (NCT02644668). [16

Novartis developed a small molecule LMI070 targeted to increase exon 7 retention in the SMN2 transcript that began enrolling infants with Type 1 SMA in an open label Phase I/II study (started in 2015). The study was halted due to preclinical toxicology. The study is ongoing to follow patients for safety but is no longer recruiting patients (NCT02268552).

Quinazolines are a family of compounds that can inhibit an enzyme involved in RNA turnover, which can subsequently increase SMN2 expression [24]. Early research on these compounds demonstrated poor blood brain barrier penetration, and further optimizations 
have led to development of a more novel compound called RG3039 which appears to improve the phenotype in SMA mice [24]. RG3039 was studied in a Phase I clinical trial to study safety and efficacy through a first in human, double blind, placebo-controlled, ascending single dose, safety and pharmacokinetics study in healthy volunteers. Further drug development into later phase clinical trials has not been pursued to date. [16

Nusinersen (IONIS SMNrx, Spinraza) is an antisense oligonucleotide (ASO) developed by Ionis and Biogen which binds to SMN2 pre-RNA and corrects splicing. It enhances the inclusion of exon 7 in SMN2 pre-mRNA, and thus promotes the increase of full-length SMN protein production. Nusinersen received a broad FDA approval for use in all subtypes of SMA (caused by mutations on chromosome 5q) in the USA on December 23, 2016 based on evidence of efficacy in a double blind controlled clinical trial of 121 patients with SMA type 1 diagnosed before the age of 7 months [23]. It is given by intrathecal administration, and the trial tested the active administration versus a sham procedure to control bias. The motor endpoints in the study included head control, sitting, kicking in supine position, rolling, crawling, standing and walking. An interim analysis of 82 patients showed motor improvement in $40 \%$ of patients on active treatment, versus none in the sham group, and the trial was interrupted early due to this efficacy signal to allow all participants to roll into an open label study extension. Additional support came from an open label single arm study assessing the efficacy/safety of presymptomatic infants with SMA (with 2 or 3 copies of SMN2) - NURTURE (NCT02386553), where interim analysis has shown very favorable results. Nusinersen (Spinraza) is now approved in the USA for all types of SMA, and has been submitted to European regulators late in 2016. [23, 25]

Gene therapy is also showing promising results in SMA. SMA mice treated with selfcomplementary adeno-associated virus-9 carrying the human SMN cDNA (scAAV9-SMN) showed widespread transgene expression in spinal cord motor neurons. This was confirmed in nonhuman primates. In mice, there was complete rescue of the disease phenotype [26]. In human trials with AVXS-101 (NCT02122952) in 15 infants with SMA type 1 aged up to 6 months, a single dose of viral AAV9 vector via intravenous delivery was administered to two cohorts: Cohort $1(\mathrm{n}=3) 6.7 \times 10 \hat{10} \mathrm{vg} / \mathrm{kg}$ of AVXS-101; Cohort $2(\mathrm{n}=12) 2.0 \times 10 \hat{10} \mathrm{vg} / \mathrm{kg}$ of AVXS-101 (clinicaltrials.gov). The outcomes were safety, survival and motor function at 13.6 months, and initial results presented at the American Academy of Neurology meeting in Boston on April 2017 showed promising preservation and improvement in motor function $[23,26,27]$.

\section{Standard of Care}

In 2007, a consensus statement for the standard of care in spinal muscular atrophy was published by Dr. Ching Wang and a group of experts in SMA (International Standard of Care Committee for Spinal Muscular Atrophy) [28]. In addition to defining the diagnostic workup for suspected SMA, this statement tried to create more uniform practices in the care of patients with SMA, given large variability in care and outcomes. These goals were centered on items such as pulmonary care, nutritional support, orthopedic considerations (especially around issues of scoliosis repair), perioperative management, and palliative care issues. In light of new therapeutics, care standards are evolving. Clinicians should test as 
early as possible for SMA when it is suspected, given that early treatment is better. An additional consideration is the potential need for newborn screening for SMA. One of the hurdles to treatment of patients with SMA is being able to recognize and diagnose them early, prior to the loss of irreplaceable motor neuron units. For SMA type 1, denervation begins within the first 3 months of life and more than $90 \%$ of motor units are lost within the first 6 months of age [29]. If newborn screening (NBS) was employed, patients could be identified and treatment initiated presymptomatically in a greater majority of patients. In order for NBS to be feasible for SMA, it must be cost-efficient, high throughout, and easy to implement in various NBS laboratories. Results should have rapid turnaround (typically this is within 5 days) [29]. After initial screening, a second-tier test would likely be needed to confirm or quantify $S M N 1$ number (deletions/mutations) and then evaluate for $S M N 2$ copy number. NBS pilot studies in the context of the initiation of rapid treatment will help define the feasibility of NBS to be broadly applied and help define the phenotype in a much larger cohort of presymptomatic infants with SMA of various subtypes.

\section{Conclusion}

Chromosome 5 linked SMA can be readily diagnosed through genetic testing. While there is currently no newborn screening, the availability of disease-targeted treatments, and the growing evidence of greater efficacy in those treated earlier may result in the establishment of newborn screening programs. Currently, more widespread prenatal screening for carrier status for SMA may also help towards testing and subsequent identification of infants in utero or immediately post-natally, allowing for earlier diagnosis.

Multidisciplinary and proactive care can improve outcomes and quality of life. Years of rigorous natural history data have set the stage for validated endpoints and robust trial designs in recent clinical trials. Another important foundation for success is the collaboration between patient groups and academic researchers. Without the active participation of patients this progress would not have been possible. Academic researchers working together nationally and internationally to develop outcome measures, care and data standards have also been critical partners. Finally, private-public partnerships and collaborations between academic and industry investigators were instrumental in advancing new therapeutics for SMA along the "pipeline".

Several trials have been completed, others are underway. These trials have resulted in the approval of the first disease-modifying drug for SMA in the USA, the ASO drug nusinersen. Recent gene therapy approaches are promising. The experience to date suggests that treatments are more effective when started early. These advances are providing promise for patients with SMA and also will pose new diagnostic and care considerations, including for example the need for newborn screening and redefining of standard of care practices.

\section{References}

Papers of particular interest, published recently, have been highlighted as:

* Of importance

** Of major importance

Curr Neurol Neurosci Rep. Author manuscript; available in PMC 2018 October 06. 
1. Kolb SJ, Kissel JT. Spinal muscular atrophy: A timely review. Arch Neurol. 2011; 68(8):979-983. [PubMed: 21482919]

* 2. Pechmann A, Kirschner J. Diagnosis and new treatment avenues in spinal muscular atrophy. Neuropediatrics. 2017 This paper gives an overview of diagnosing SMA as well as reviews standard of care practices and provides a timeline of the development of therapeutics for SMA.

3. Wen-Yo T, Simpson JE, Highley JR, Heath PR. Spinal muscular atrophy: Factors that modulate motor neurone vulnerability. Neurobiology of disease. 2017; 102:11-20. [PubMed: 28161391]

* 4. Burghes AHM, Beattie CE. Spinal muscular atrophy : why do low levels of survival motor neuron protein make motor neurons sick ? Nature Reviews. 2009; 10:597-609. The author provides a detailed review of the function of SMN and discusses hypotheses for why low SMN leads to SMA and why it targets motor neurons.

5. Arnold WD, Kassar D, Kissel JT. Spinal muscular atrophy : Diagnosis and management in a new therapeutic era. Muscle Nerve. 2015; 51:157-167. [PubMed: 25346245]

6. Grotto S, Cuisset JM, Marret S, et al. Type 0 spinal muscular atrophy: further delineation of prenatal and postnatal features in 16 patients. J Neuromuscul Dis. 2016; 3:487-495. [PubMed: 27911332]

7. Pera MC, Coratti G, Forcina N, et al. Content validity and clinical meaningfulness of the HFMSE in spinal muscular atrophy. BMC Neurology. 2017; 17(39):1-10. [PubMed: 28056870]

* 8. Bishop KM, Montes J, Finkel RS. Motor milestone assessment of infants with spinal muscular atrophy using the Hammersmith infant neurological exam - part 2: Experience from a nusinersen clinical study. Muscle Nerve. 2017:1-4. The authors discuss the reliability and feasibility of the use of the HINE-2 scale for assessing infants with SMA in response to therapeutics and in clinical trials.

9. Iannaccone ST. Outcome measures for pediatric spinal muscular atrophy. Arch Neurol. 2002; 59:1445-1450. [PubMed: 12223032]

10. Seferian AM, Moraux A, Canal A, et al. Upper limb evaluation and one-year follow up of nonambulant patients with spinal muscular atrophy: an observational multicenter trial. PLOS ONE. 2015; 10(4):e021799.

11. Bonati U, Holiga S, Hellback N, et al. Longitudinal characterization of biomarkers for spinal muscular atrophy. Ann Clin Transl Neurol. 2017; 4(5):292-304. [PubMed: 28491897]

12. Oskoui M, Levy G, Garland CJ, et al. The changing natural history of spinal muscular atrophy type 1. Neurology. 2007; 69(20):1931-6. [PubMed: 17998484]

13. Finkel RS, McDermott MP, Kaufmann P, et al. Observational study of spinal muscular atrophy type I and implications for clinical trials. Neurology. 2014; 83(9):810-7. [PubMed: 25080519]

14. Kaufmann P, McDermott MP, Darras BT, et al. Prospective cohort study of spinal muscular atrophy types 2 and 3. Neurology. 2012; 79(18):1889-97. [PubMed: 23077013]

* 15. Mercuri E, Finkel R, Montes J, et al. Patterns of disease progression in type 2 and 3 SMA: Implications for clinical trials. Neuromuscul Disord. 2016; 26:126-131. This paper reports on one of the largest sets of longitudinal data in ambulant and nonambulant patients with type 2 and type 3 SMA and describes the change in the Hammersmith Functional motor scale over time. [PubMed: 26776503]

** 16. Calder AN, Androphy EJ, Hodgetts KJ. Small molecules in development for the treatment of spinal muscular atrophy. J Med Chem. 2016; 59:10067-10083. This paper provides an overview of prior and novel small molecule compounds and reviews their biochemical properties, mode of action, and for previously tested repurposed drugs provides a review of the trial data for those compounds. [PubMed: 27490705]

* 17. Farrar MA, Park SB, Vucic S, et al. Emerging therapies and challenges in spinal muscular atrophy. Ann Neurol. 2017; 81:355-368. This paper provides a review of novel therapies for SMA, and provides useful figures and tables that highlight the mechanism and targeted site of action for these drugs. [PubMed: 28026041]

18. Swoboda KJ, Scott CB, Crawford TO, et al. SMA CARNI-VAL trial part I: double-blind, randomized, placebo-controlled trial of $\mathrm{L}$-carnitine and valproic acid in spinal muscular atrophy. PLoS One. 2010; 5(8):e12140. [PubMed: 20808854] 
19. Kissel JT, Scott CB, Reyna SP, et al. SMA CARNIVAL Trial Part II: a prospective, single-armed trial of L-carnitine and valproic acid in ambulatory children with spinal muscular atrophy. PLoS One. 2011; 6(7):e21296. [PubMed: 21754985]

20. Wadman RI, Bosboom WM, van den Berg LH, et al. Drug treatment for spinal muscular atrophy type I. Cochrane Database Syst Rev. 2011; 12:CD006281.

21. Wadman RI, Bosboom WM, van den Berg LH, et al. Drug treatment for spinal muscular atrophy types II and III. Cochrane Database Syst Rev. 2011; 12:CD006282.

22. Bertini E, Dessaud E, Mercuri E, et al. Safety and efficacy of olesoxime in patients with type 2 or non-ambulatory type 3 spinal muscular atrophy: a randomised, double-blind, placebo-controlled phase 2 trial.

** 23. Scoto M, Finkel RS, Mercuri E, Muntoni F. Therapeutic approaches for spinal muscular atrophy (SMA). Gene Therapy. 2017 Epub ahead of print. This article provides a timely review of therapies for SMA including a focus on recent clinical trials and novel advances including gene replacement.

24. Zanetta C, Nizzardo M, Simone C, et al. Molecular therapeutic strategies for spinal muscular atrophy: current and future clinical trials. Clinical Therapeutics. 2014; 36(1):128-140. [PubMed: 24360800]

** 25. Finkel RS, Chiriboga CA, Vajsar J, et al. Treatment of infantile-onset spinal muscular atrophy with nusinersen: a phase 2, open-label, dose-escalation study. 2016; 388:3017-3026. This paper provides data on the use of nusinersen with type I SMA and reviews safety, motor functional, and electrophysiologic outcome measures as well as critical biodistribution and histologic data that was obtained from autopsy of three patients.

26. Meyer K, Ferraiuolo L, Schmelzer L, et al. Improving single injection CSF delivery of AAV9mediated gene therapy for SMA: a dose-response study in mice and nonhuman primates. Mol Ther. 2015; 23:477-87. [PubMed: 25358252]

27. [accessed June 21, 2017] https://www.medpagetoday.com/meetingcoverage/aan/64817

** 28. Wang CH, Finkel RS, Bertini ES, et al. Consensus statement for standard of care in spinal muscular atrophy. J Child Neurol. 2007; 22(8):1027-1049. This paper has led to a shift in clinical practices in order to improve standards of care and overall health for patients with SMA over the past ten years. [PubMed: 17761659]

29. Phan HC, Taylor JL, Hannon H, et al. Newborn screening for spinal muscular atrophy: anticipating an imminent need. Semin Perinat. 2015; 39:217-229. 


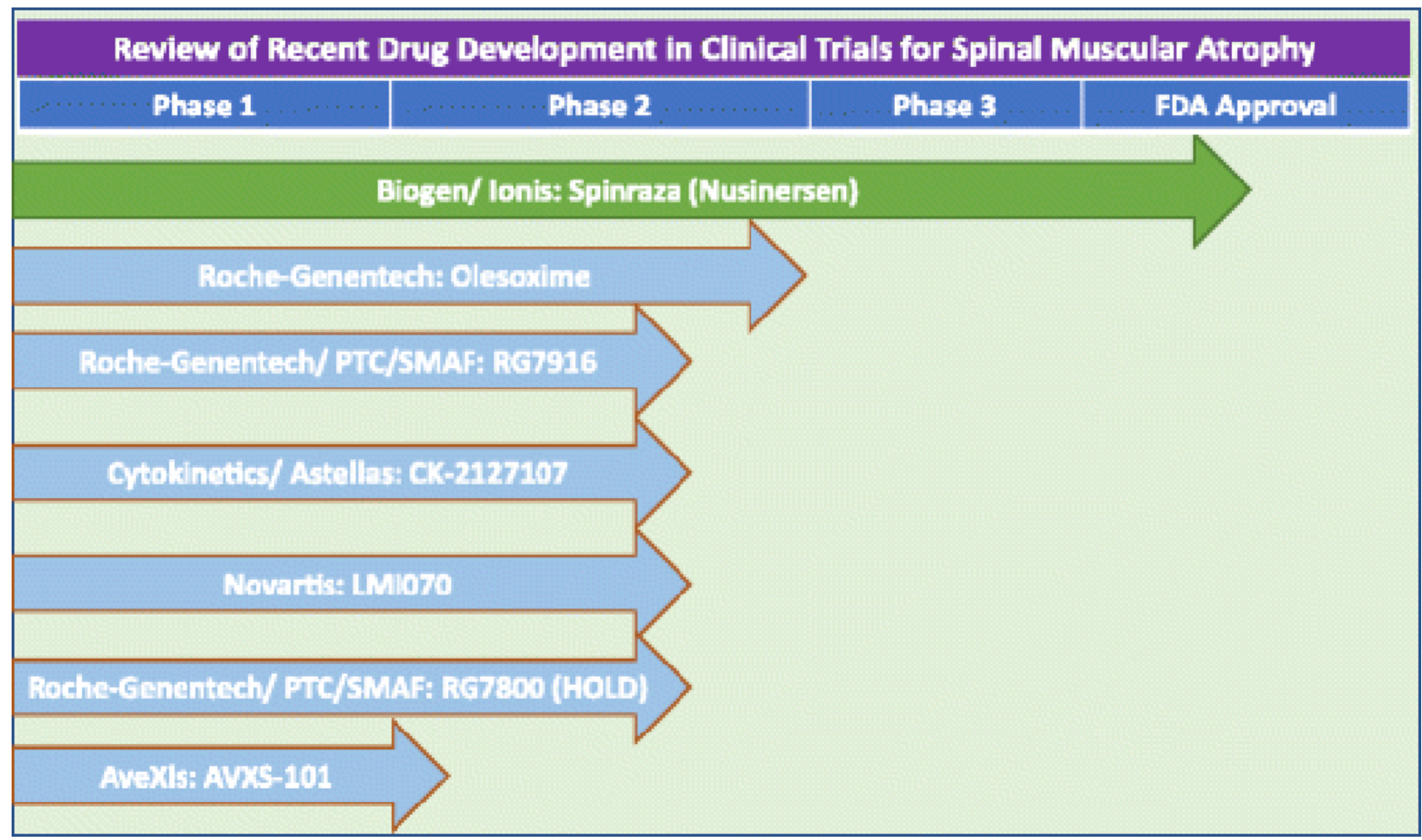

Figure 1.

References: 23, 24, and modified from www.curesma.org 


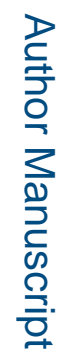
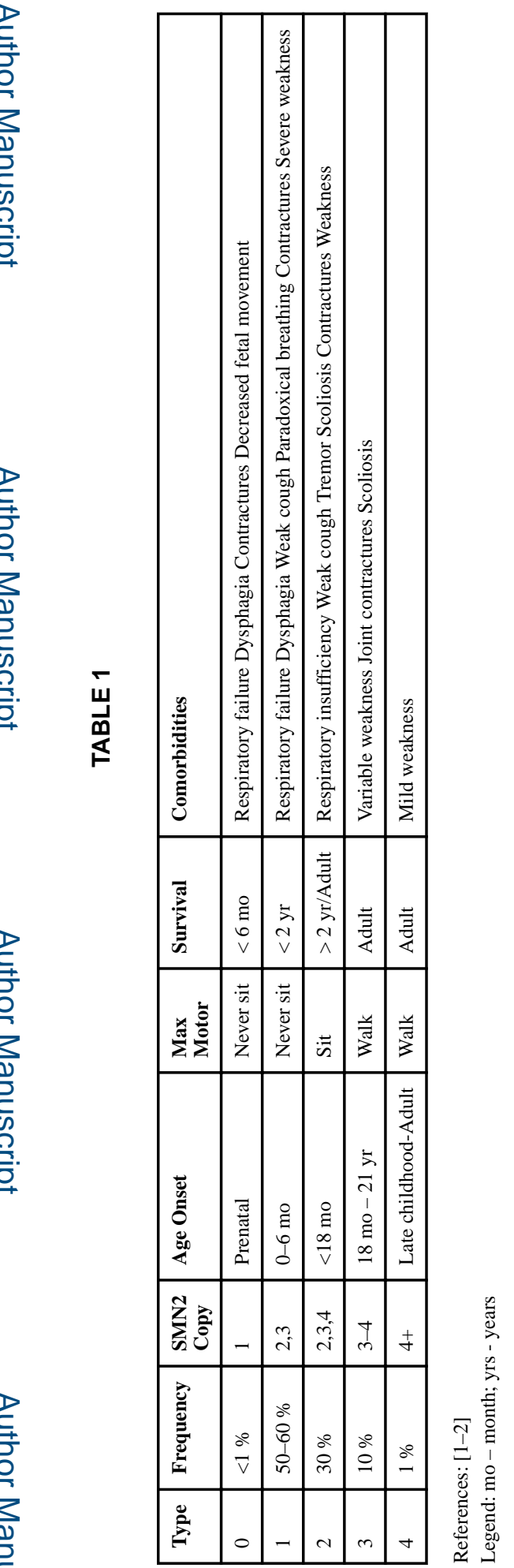

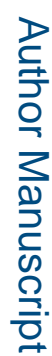

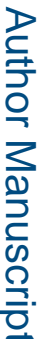

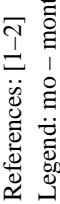

Curr Neurol Neurosci Rep. Author manuscript; available in PMC 2018 October 06. 